\title{
ARTICLES
}

\section{Long-term operation of the Rome "Explorer" cryogenic gravitational wave detector}

\author{
P. Astone ${ }^{\mathrm{a}}{ }^{\mathrm{M}}$ M. Bassan, ${ }^{\mathrm{a}, \mathrm{b}}$ P. Bonifazi, ${ }^{\mathrm{a}, \mathrm{c}}$ P. Carelli, ${ }^{\mathrm{a}, \mathrm{e}}$ M. G. Castellano, ${ }^{\mathrm{f}}$ G. Cavallari, ${ }^{\mathrm{g}}$ \\ E. Coccia, ${ }^{\mathrm{a}, \mathrm{b}}$ C. Cosmelli,,${ }^{\mathrm{a}, \mathrm{d}}$ V. Fafone, ${ }^{\mathrm{a}, \mathrm{b}}$ S. Frasca, ${ }^{\mathrm{a}, \mathrm{d}}$ E. Majorana, ${ }^{\mathrm{a}, \mathrm{h}}$ I. Modena, ${ }^{\mathrm{a}, \mathrm{b}}$ \\ G. V. Pallottino, ${ }^{\text {a,d }}$ G. Pizzella, ${ }^{\text {a,b }}$ P. Rapagnani, ${ }^{\text {a,d }}$ F. Ricci, ${ }^{\text {a,d }}$ and M. Visco ${ }^{\text {a,c }}$ \\ ${ }^{a}$ Istituto Nazionale di Fisica Nucleare, Sezioni di Roma, Roma, Italy \\ 'Dipartimento di Fisica, Università di Roma "Tor Vergata," Roma, Italy \\ ${ }^{\mathrm{c}}$ Istituto di Fisica dello Spazio Interplanetario, Consiglio Nazionale delle Ricerche, Frascati, Italy \\ ${ }^{\mathrm{d}}$ Dipartimento di Fisica, Università di Roma "La Sapienza," Roma, Italy \\ 'Dipartimento di Energetica, Università dell' Aquila, Roio Poggio, Italy \\ ${ }_{\mathrm{f}}^{\mathrm{f}}$ Istituto di Elettronica dello Stato Solido, Consiglio Nazionale delle Ricerche, Roma, Italy \\ ${ }^{\mathrm{g}} \mathrm{CER} N$, Geneva, Switzerland \\ ${ }^{\mathrm{h}}$ Dipartimento di Fisica, Università di Catania, Catania, Italy
}

(Received 12 June 1992)

\begin{abstract}
We describe the cryogenic resonant gravitational wave detector of the Rome group, named Explorer, and report on its long term operation with sensitivity for short bursts in the range $h \simeq 7-10 \times 10^{-19}$. Explorer has mass $M=2270 \mathrm{~kg}$ and is equipped with a resonant capacitive transducer followed by a dc superconducting quantum interference device amplifier. It has been operated at $T \simeq 2.6 \mathrm{~K}$ in a cryostat cooled with superfluid helium. With a transducer voltage bias of $320 \mathrm{~V}(E=6.15 \mathrm{MV} / \mathrm{m})$ the two resonant modes have frequencies of 904.7 and $921.3 \mathrm{~Hz}$ with coupled quality factors, respectively, of $0.77 \times 10^{6}$ and $1.0 \times 10^{6}$. The description of the experimental apparatus and of its calibration is followed by the analysis of the noise and the calculation of the expected sensitivity of the detector: $h \simeq 8 \times 10^{-19}$ (under the assumption of bursts with duration of $1 \mathrm{~ms}$ ). We then describe the data acquisition system and the techniques of data analysis, discussing the filtering algorithms. The last section reports the experimental results obtained during the operation of the detector from May 1990 to December 1991. During this period the data were recorded for more than two-thirds of the total time: we show the distributions of the data and the hourly averages of the sensitivity. The data taken from May 1991 to December 1991 have also been used to establish a new improved upper limit for the rate and strength of gravitational wave pulses; at $h=2 \times 10^{-17}$, for example, there are no more than 0.5 events/day as averaged over a period of 134 days.
\end{abstract}

PACS number(s): $04.80 .+\mathrm{z}$

\section{INTRODUCTION}

The basic problems of the gravitational wave (GW) detection experiments are (a) how to obtain the sensitivity required for detecting collapses occurring at a rate of practical interest (such as those from the Virgo cluster, with $h \sim 10^{-21}$ and a rate of about 1 event/month [1]), and (b) how to operate the detectors continuously with good performance. We consider here resonant bar antennas, the only detectors that have been so far operated for substantial amounts of time (several months) at sensitivities in the range below $h=10^{-18}$.

As regards problem (a), the classical cross-section theory, which assumes the antenna be a single large-mass quadrupole, relates the minimum detectable Fourier transform $H$ of the metric tensor perturbation at the bar resonance frequency to the effective noise temperature for burst detection, $T_{\text {eff }}$, which represents the total-energy fluctuations of the observed antenna modes after proper filtering of the data:

$$
H=\frac{L}{v_{s}^{2}}\left(\frac{k T_{\mathrm{eff}}}{M}\right)^{1 / 2},
$$

where $L$ and $M$ are the length and mass of the bar, supposed to be cylindrical, and $v_{s}=5400 \mathrm{~m} / \mathrm{s}$ is the velocity of sound in the bar $[2,13]$.

It has been shown [3] that the minimum effective temperature that can be obtained with linear amplifiers (i.e., without resorting to quantum evading schemes [4]) has a limit given by twice the noise temperature $T_{n}$ of the amplifier. With the dc superconducting quantum interference device (SQUID) amplifiers used at present, whose noise temperature is estimated to be lower than $10^{-6} \mathrm{~K}$ at $1000 \mathrm{~Hz}$, a resonant antenna with a mass of a few tons should reach the sensitivity of $h \sim 10^{-21}$.

However, the Giffard limit $T_{\text {eff }}=2 T_{n}$ can actually be achieved only if two matching conditions are satisfied [5]: First, the amplifier must be properly impedance matched; second, the energy-transfer efficiency of the transducer 
and the quality factors of the oscillators (the $\beta$ and $Q$ factors) must be sufficiently large and the operating temperature of the antenna must be small.

This is the goal of the new generation of cryogenic detectors to be operated below $100 \mathrm{mK}$ : One detector is being built at Stanford [6]; another one, called Nautilus, has been built and tested below $100 \mathrm{mK}$ by the Rome group [7] and will be put in operation in the next months. Another detector similar to Nautilus is now being built at the Legnaro laboratory near Padova.

As regards problem (b), long-term operations of cryogenic detectors have been reported by the groups of Stanford [8], Rome [2] and Louisiana State University (LSU) [9]. In all cases, however, while the average values of the noise were good (but still far from that needed to observe the Virgo cluster), i.e., close to the design values (with $T_{\text {eff }}$ in the range $10-20 \mathrm{mK}$ ), the observational results were not as satisfactory as expected because of the occasional presence of excess noise [10].

As regards the Explorer detector, its performance has been greatly improved in the last years, especially as the excess noise is concerned, by operating it with the helium in the superfluid regime [2]. This is obtained by pumping on the liquid-helium bath that keeps the detector cool: The temperature is reduced from 4.2 to $2 \mathrm{~K}$, and more important, the cryogenic system becomes much quieter, probably because below the $\lambda$ point the vibrations due to the boiling of the liquid He disappear [11].

\section{EXPLORER DETECTOR}

The Explorer detector $[12,13]$ consists of a resonant bar equipped with a resonant capacitive transducer and a dc SQUID amplifier. It is located in the CERN laboratories $(6.1 \mathrm{E}, 46.2 \mathrm{~N})$ and in February 1990 it was oriented to an azimuthal angle of $39.3^{\circ}$, approximately parallel to the Louisiana State University cryogenic detector, in order to improve the detection capability of coincidence experiments.

The bar is a cylinder $(60 \mathrm{~cm}$ in diameter, $297 \mathrm{~cm}$ in length) made of Al 5056 (a low-dissipation Al-Mg alloy) with mass $M=2270 \mathrm{~kg}$ and resonance frequency (first longitudinal mode) $v_{b}=915.69 \mathrm{~Hz}$ at the liquid-helium temperature. This bar, with its superconducting instrumentation, is housed in a cryostat designed for operation at $4.2 \mathrm{~K}$, which is now functioning very satisfactorily at $2 \mathrm{~K}$.

\section{A. Cryostat and the vibration-isolation system}

The cryostat (Fig. 1) consists of five horizontal cylinders [14]. The external one is a steel vacuum enclosure. The second one is the liquid-nitrogen container (460 liters). The third cylinder is a thermal shield cooled near $30 \mathrm{~K}$ by the gas evaporated from the liquid-helium container. This container, consisting of the space between the fourth and fifth cylinder, has a capacity of 3300 liters and is made of stainless steel; its ends are closed by large flanges $(1.7 \mathrm{~m}$ of diameter) with indium seals (probably the largest $\lambda$ tight flanges).

In the past, with the cryostat operating at $4.2 \mathrm{~K}$, the liquid-helium consumption was 60 liters/day. At present, we pump on the liquid-helium bath down to a pressure of 15 mbar, thereby obtaining a bath temperature of $1.8 \mathrm{~K}$, below the $\lambda$ point $(2.17 \mathrm{~K})$, without any problem from the flanges. This has several advantages, in addition to reducing the temperature: We no longer have the acoustic noise due to the boil off of the helium and to the thermal oscillations of the gas in the vessels, and the liquid-helium consumption is now reduced to 35 liters/day.

The temperature $T$ of the bar, which is measured with a germanium thermometer, is not, in general, equal to that of the helium bath because the heat link is very weak. Its value depends on the balance between the thermal inputs and the cooling effect of the residual gas in the experimental space. If the pressure is reduced below $10^{-7}$ mbar, the bar temperature eventually drifts a few kelvin above the bath. At pressures larger than $10^{-6}$ mbar, the heating effect is effectively countered by the heat link to the bath. Since December 1990 we have succeeded in maintaining the antenna temperature stable at $T \simeq 2.6 \mathrm{~K}$ with an amount of exchange gas (about $10^{-6}$ mbar) that has no measurable influence on both the mechanical $Q$ 's and the transducer electrical breakdown limit.

The isolation of the bar from external acoustic and seismic disturbances is provided by a system of low-pass mechanical filters in cascade, with a total attention of 210 $\mathrm{dB}$ at the observed frequencies $(\sim 900 \mathrm{~Hz})$.

The first stage of filtering consists of the liquid-helium container suspended with four titanium rods hanging from mechanical dampers (stacks of steel and rubber disks) at room temperature. Particularly important are the following stages, which were designed to provide the large attenuation required also for isolating the bar from the boiling noise of the helium. The bar is suspended with a titanium cable $(2 \mathrm{~m}$ long and $5 \mathrm{~mm}$ in diameter) wrapped around the central section of the bar. The ends of this cable are fastened to a steel ring of $1000 \mathrm{~kg}$ mass, which is in turn suspended by four cables to another massive ring $(1000 \mathrm{~kg})$ made of bronze. This rests on a fourcantilever-beam suspension on the bottom of the liquidhelium container.

\section{B. Transducer and the dc SQUID}

The vibrations of the bar are converted into electrical signals by a capacitive transducer, resonating at the antenna frequency in order to improve the energy transfer from the bar to the electronics. The signals are applied to the input coil of a dc SQUID amplifier by means of a superconducting transformer, which provides the required impedance matching.

The capacitive transducer [15] bolted to one end of the antenna, consists of a vibrating disk with mass $M_{t}=0.40$ $\mathrm{kg}$ (effective mass $m_{t}=0.340 \mathrm{~kg}$ ) and of a fixed plate with a gap $d=52 \mu \mathrm{m}$ and a total capacitance $C_{t}=3890 \mathrm{pF}$.

The transducer and bar form a system of two oscillators, which are well coupled since the frequency $v_{t}$ of the lowest symmetrical flexural mode of the transducer is very close to that of the first longitudinal mode of the 


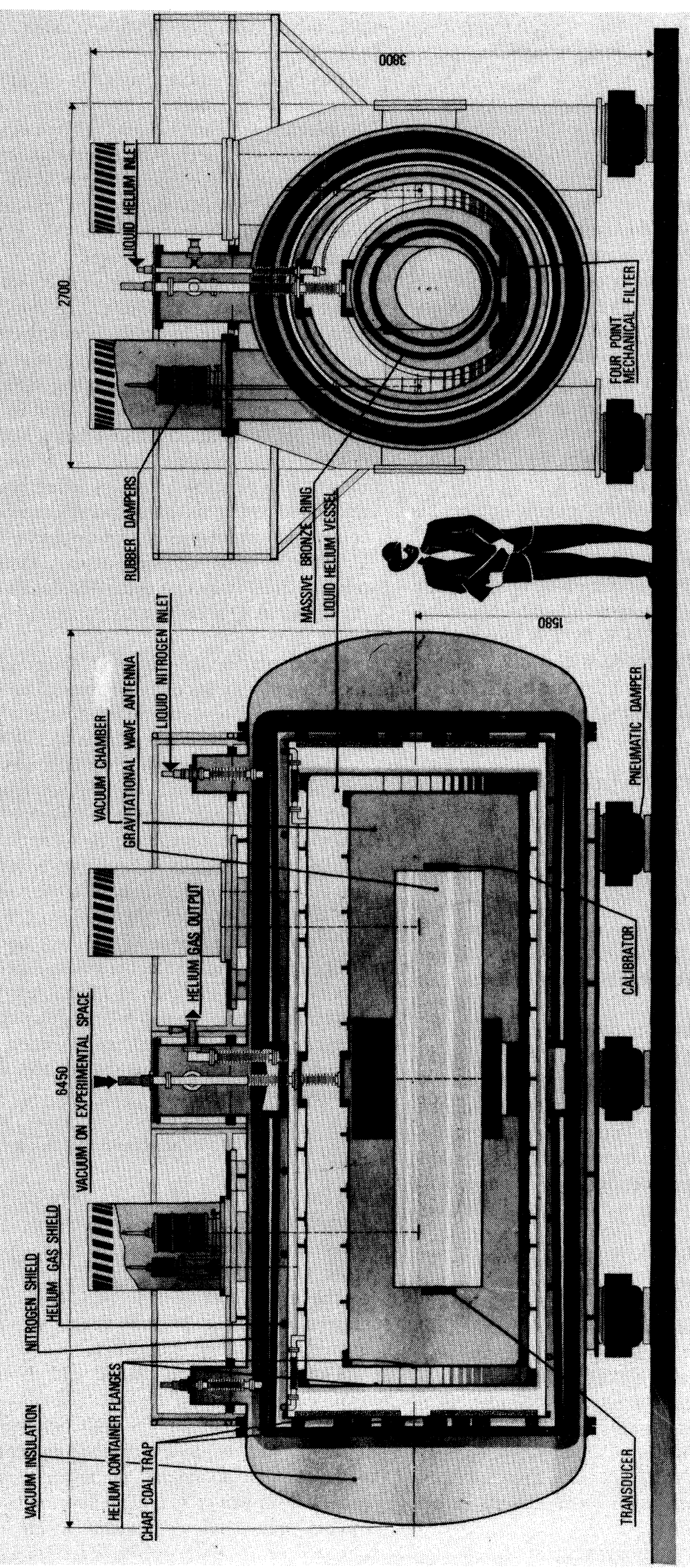

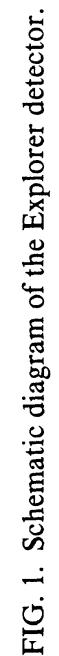


bar. More precisely, $v_{t}$ depends on the bias voltage $V_{b}$ of the transducer according to the relation [16]

$$
v_{t}=\left(v_{t_{0}}^{2}-c V_{b}^{2}\right)^{1 / 2} \text {, }
$$

where $v_{t_{0}}=917.95 \mathrm{~Hz}$ and $c=0.125 \mathrm{~Hz}^{2} / \mathrm{V}^{2}$.

The frequencies $\left(v_{-}\right.$and $\left.v_{+}\right)$of the resulting normal modes are spaced of about $\nu_{b} \sqrt{\mu}$, where $\mu=3.031 \times 10^{-4}$ is the ratio between the effective masses of the transducer disk and bar. With $V_{b}=320 \mathrm{~V}$, we have $v_{-}=904.7 \mathrm{~Hz}$ and $v_{+}=921.3 \mathrm{~Hz}$.

Here we have an amplification effect from the vibration amplitude $x(t)$ of the end face of the antenna to that $y(t)$ of the transducer: Since the energy is almost totally transferred between the two oscillators, because of their good coupling, this amplification is $\sim 1 / \sqrt{\mu}$.

The transduction constant between disk vibrations (relative to the bar end) and output voltage $v(t)$ is proportional to the field $E=V_{b} / d$, where $V_{b}$ is the dc applied voltage:

$$
\alpha=\frac{v(t)}{y(t)-x(t)}=\gamma_{t} E
$$

where $\gamma_{t}=m_{t} / M_{t}=0.85$ is a geometrical factor determined by the mode shape of the transducer vibration [16].

The high-impedance transducer is connected to the low-inductance $\left(L_{\text {in }}=1 \mu \mathrm{H}\right)$ input coil of the SQUID through a decoupling capacitance $\left(C_{d}=100 \mathrm{nF}\right)$ and a superconducting transformer with high turn ratio $(N=1270)$, primary inductance $L_{0}=2.5 \mathrm{H}$, secondary inductance $L_{s}=1.6 \mu \mathrm{H}$, and coupling factor $k_{t}=0.77$. The impedance of the transducer circuit is

$$
Z(\omega)=\frac{1}{j \omega C}+j \omega L_{0}\left[1-k_{t}^{2} \frac{L_{s}}{L_{\mathrm{in}}+L_{s}}\right]+r
$$

where $r$ represents the losses of the various elements and $C$ the series connection of $C_{t}$ and $C_{d}$. This circuit exhibits a resonance at $v_{\mathrm{el}}=1940 \mathrm{~Hz}$ : We have therefore a third (electrical) mode, which is, however, only weakly coupled to the mechanical modes (its effect on the values of the frequencies $v_{-}$and $v_{+}$is very small, of the order of $1 \mathrm{mHz}$ ).

The dc SQUID is a planar device with a multiloop geometry that exhibits very low intrinsic noise and good coupling to the external world [17]. The SQUID is biased with a dc current $(42 \mu \mathrm{A})$ and an ac modulation flux at $v_{m}=80 \mathrm{kHz}$ applied through a coil. Its output signal is applied to a low-noise differential field effect transistor (FET) amplifier [18] through a cooled $L C$ resonant circuit $(Q=70)$ tuned at $v_{m}$ (tank circuit), which provides the proper noise impedance matching. The SQUID is operated in the usual flux-locked configuration [19].

The FET amplifier is followed by a lock-in amplifier, driven at $v_{m}$, whose output $V(t)$ is fed back to the SQUID via a weakly coupled coil in order to stabilize its operating point and to linearize its input-output $(V / \phi)$ characteristics.

The feedback factor, which depends on the gain of the lock-in amplifier, is substantial at very low frequencies $(\sim 40 \mathrm{~dB})$, almost negligible in our observation range because of the attenuation introduced by the integration time constant $\left(t_{0}=0.3 \mathrm{~s}\right)$ of the lock-in amplifier. However, the feedback coil, in addition to directly applying flux to the SQUID loop, exhibits some backward cou-
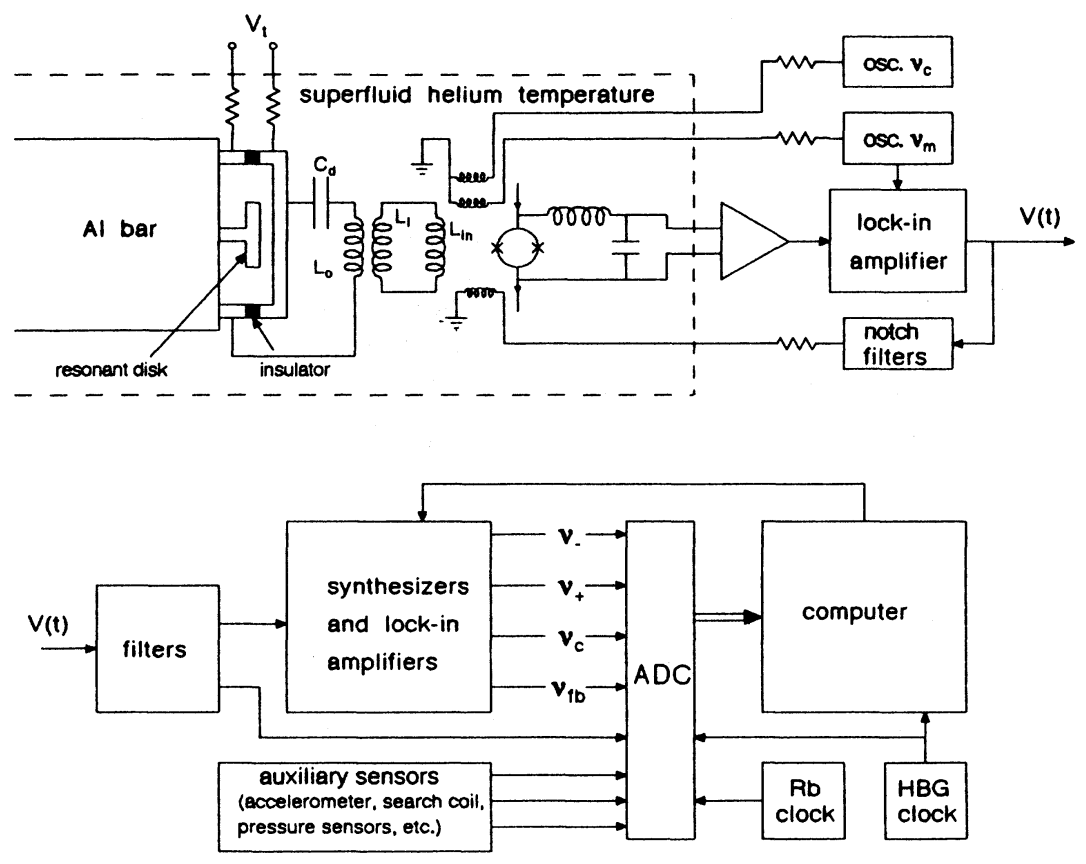

FIG. 2. Block diagram of the experimental apparatus. 
pling [20] to the SQUID input coil $L_{\text {in }}$ and therefore to the high- $Q$ mechanical modes (through the superconducting transformer and transducer). We avoid any unwanted regeneration or degeneration effect (which would modify the $Q$ values of the modes) by inserting in the feedback network two notch circuits in cascade (one tuned at $v_{-}$, the other at $v_{+}$) providing sharp attenuation $\left(\sim 10^{-3}\right)$ at the mode frequencies.

The gain of the SQUID amplifier, more precisely the slope of its $V / \phi$ characteristics, depends on the operating point of the device as well as on the temperature and $\mathrm{rf}$ noise reaching the SQUID: It occasionally exhibits some variations due to the switching between two stable operating points, the maximum and minimum of the $V / \phi$ characteristics, that have different gain. For this reason we continuously monitor the SQUID operation by means of a fixed-amplitude ac magnetic flux $\left(\phi_{c}=1.3 \times 10^{-5} \phi_{0}\right)$ at a frequency $v_{c}(913 \mathrm{~Hz}$, that is, between the frequencies of the two modes), which is applied to the SQUID through a third coil. The corresponding output signal is used to normalize the data during the analysis.

The output signal from the SQUID instrumentation is sent to four lock-in amplifiers, which demodulate it at the frequencies of the modes $\left(v_{-}\right.$and $\left.v_{+}\right)$, at the frequency of the calibration flux $\left(v_{c}\right)$ and at $v_{\mathrm{FB}}=909 \mathrm{~Hz}$. The last one provides information on the wideband noise of the detector in the region between the modes.

The experimental apparatus includes a vibration sensor (accelerometer) and a magnetic-field sensor (search coil), both located on the cryostat. These sensors monitor the environment of the laboratory; their output signals may be used as a veto. Another signal, which is recorded to be used as a veto, is the output of the antenna seen through the SQUID instrumentation and bandpass filtered between 20 and $70 \mathrm{~Hz}$, i.e., in the range of the resonance frequencies of the mechanical filters used in the suspension of the antenna.

The block diagram of the experimental apparatus is shown in Fig. 2.

\section{SIGNAL, NOISE, AND SENSITIVITY}

\section{A. Signal}

We consider now the predicted response of the detector to a short burst of gravitational radiation that deposits a given amount of energy $E_{s}$ in the first longitudinal mode of the uncoupled bar (assumed at rest). The interaction of the GW burst with the transducer can be ignored because of its negligible mass quadrupole moment. When using a resonant transducer, this energy is divided into equal parts in the two modes if the coupling of the two oscillators is perfect $\left(v_{t}=v_{b}\right)$; otherwise, the larger part of $E_{s}$ is deposited in the mode where the antenna oscillator is dominant.

The energy coupling factor, which is the fraction of the energy $E_{s}$ that is available for the measurement as an electrical signal in the transducer (in open-circuit conditions) is $\beta_{c \pm}$ for each of the two modes:

$$
\beta_{c \pm}=\frac{\frac{1}{2} C_{b} V_{ \pm}^{2}}{E_{s}}=\frac{\alpha^{2} C_{t}}{2 m_{t \pm} \omega_{ \pm}^{2}},
$$

where $m_{t \pm}$ are the effective masses of the transducer for the two modes [16,21]. The total energy available is $\beta_{c} E_{s}$, where

$$
\beta_{c}=\beta_{c-}+\beta_{c+} \simeq \frac{\alpha^{2} C_{t}}{m_{t} \omega_{b}^{2}},
$$

with good approximation even if the coupling between the two oscillators is not perfect.

The energy coupling factor, in terms of the electrical energy available in the SQUID input coil, for each of the modes is

$$
\beta_{L \pm}=\frac{\frac{1}{2} L_{\mathrm{in}} I_{\mathrm{in}}^{2}}{E_{s}} \simeq \frac{\beta_{c \pm} L_{\mathrm{in}} N_{e}^{2}}{C_{t}\left|Z\left(\omega_{ \pm}\right)\right|^{2}},
$$

where $N_{e}=N k_{t} L_{s} /\left(L_{s}+L_{\text {in }}\right)$ represents the effective transformation ratio of the transformer and the formula is valid if $v_{e} \neq v_{b}, v_{t}$. In our detector, when the transducer is biased at $V_{b}=320 \mathrm{~V}(E=6.15 \mathrm{MV} / \mathrm{m})$, we have

$$
\beta_{L-}=1.6 \times 10^{-4}, \quad \beta_{L+}=2.2 \times 10^{-4} \text {. }
$$

The shape of the expected signals [see Eq. (10) below] as well as the spectrum of the noise and consequently the sensitivity of the detector are determined by the merit factors $Q_{ \pm}$of the modes, which are in turn affected by the energy coupling factor.

In the absence of coupling between the electrical and mechanical parts of the detector (that is, when $V_{b}=0$ ), the $Q$ 's of the modes, denoted with $Q_{0 \pm}$, are given by a combination of those of the bar $\left(Q_{b}\right)$ and transducer $\left(Q_{t}\right)$ oscillators. In particular, with perfect tuning $\left(v_{t}=v_{b}\right)$, we have

$$
Q_{0-}^{-1}=Q_{0+}^{-1}=\frac{Q_{b}^{-1}+Q_{t}^{-1}}{2}
$$

These $Q$ 's are determined by the intrinsic losses of the aluminum alloy and by the dissipations contributed by the surface machining and the fastening of the transducer to the bar.

When the transducer is biased, the losses of the electrical circuit also contribute to the $Q$ 's of the modes, according to the approximate law

$$
Q_{ \pm}^{-1} \simeq Q_{0 \pm}^{-1}+\beta_{c \pm} v_{ \pm}\left(Q_{\mathrm{el}} v_{\mathrm{el}}\right)^{-1}
$$

$Q_{\mathrm{el}}=\left(2 \pi v_{\mathrm{el}} r C\right)^{-1}$ (usually quite smaller than $Q_{0 \pm}$ ) is the merit factor of the electrical circuit. We have $Q_{\mathrm{el}} \sim 4 \times 10^{4}$ and, with $V_{b}=320 \mathrm{~V}, Q_{-}=0.77 \times 10^{6}$, $Q_{+}=1.01 \times 10^{6}$.

We measured the $Q$ factor of the modes in several occasions at various voltages applied to the transducer. These results are summarized in Fig. 3, a plot of the average inverse $Q$ versus the square of the transducer voltage, which is in good agreement with the law of Eq. (9). From this plot we deduce $Q_{0_{-}}=Q_{0+} \simeq 6.4 \times 10^{6}$.

At the output of the SQUID instrumentation, at each of the two modes, the response to a short burst of gravita- 


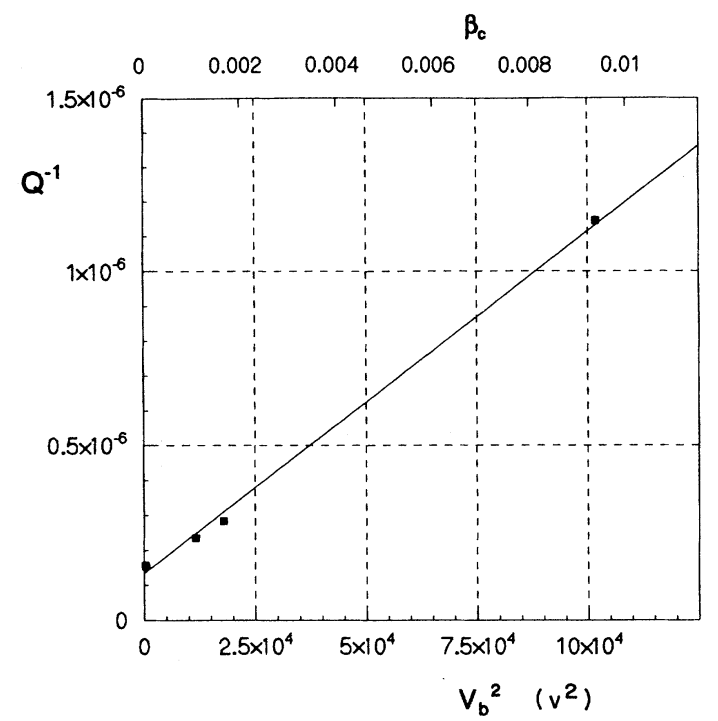

FIG. 3. Experimental values of $Q^{-1}=\frac{1}{2}\left(Q_{-}^{-1}+Q_{+}^{-1}\right)$ vs the square of the transducer voltage.

tional radiation occurring at $t=0$ (with power spectral density constant in the frequency band including $v_{-}$and $\left.v_{+}\right)$is

$$
v_{ \pm}(t)=V_{ \pm} \exp \left[\frac{-t}{\tau_{ \pm}}\right] \sin \left(\omega_{ \pm} t\right),
$$

where the coefficients $V_{ \pm}$have similar values (even if the transducer is not perfectly tuned to the bar) and

$$
\tau_{ \pm}=\frac{Q_{ \pm}}{\pi v_{ \pm}}
$$

The use of a lock-in amplifier at each frequency $v_{ \pm}$allows us to demodulate the time evolution of the amplitude of $v_{ \pm}(t)$ in a bandwidth defined by the integration time. As the phase of the incoming signal is unknown, both the outputs of a two-phase lock-in amplifier are needed to reconstruct the signal amplitude: In the following we shall refer to them as $p(t)$ and $q(t)$ ("in-phase" and "quadrature" components with respect to the reference). The signal energy is then proportional to

$$
r_{ \pm}^{2}(t)=p_{ \pm}^{2}(t)+q_{ \pm}^{2}(t) ;
$$

the amplitude is proportional to the square root of this, while the absolute-phase information is lost.

The antenna state of motion, for each of the two modes, is often represented in a Cartesian $p, q$ plane, where a conversion to polar coordinates yields the vibration amplitude and phase relative to the reference.

The integration time, finally, affects the leading edge of the signal wave form.

\section{B. Noise}

The fundamental sources of noise in the detector are the Brownian motion of the mechanical oscillators (that we assume in equilibrium at the thermodynamical tem- perature $T$ ) and the wideband noise of the SQUID and its associated instrumentation (including the losses of the transformer and tank circuit, and the noise of the FET amplifier).

In our detector the effect of back action, due to the noise current flowing in the transducer and "heating" the modes (i.e., providing an additional noise force that drives the oscillators), is negligible. The current arising from the voltage noise of the SQUID amplifier (whose power spectrum is estimated to be lower than $10^{-30}$ $\mathrm{V}^{2} / \mathrm{Hz}$ ) is nonmeasurable; the contribution of the losses of the electrical circuit is negligible as a consequence of the values of the energy coupling factor $\beta_{c}\left(\sim 10^{-2}\right)$ and the $Q$ factor of the electrical circuit $\left(Q_{\mathrm{el}} \sim 4 \times 10^{4}\right)$.

Since the Brownian noise is a narrow-band process, it is best characterized, for each mode, in terms of the total variance or rms value (standard deviation).

The rms amplitude of the normal modes, as expected from the theory of Brownian noise, is

$$
y_{\mathrm{Br} \pm}=\left(\frac{k T}{m_{t \pm} \omega_{ \pm}^{2}}\right)^{1 / 2},
$$

where $m_{t \pm}$ are the effective masses of each mode, which are both equal to $2 m_{t}$ if the coupling is perfect $\left(v_{b}=v_{t}\right)$.

These vibrations generate a current $I_{\mathrm{Br} \pm}$ in the readout circuit that couples into the SQUID a magnetic flux [13]:

$$
\phi_{\mathrm{Br} \pm}=M I_{\mathrm{Br} \pm}=\frac{k_{c} M N}{2\left|Z\left(\omega_{ \pm}\right)\right|} \gamma_{t} E y_{\mathrm{Br} \pm} \text {. }
$$

We remark that the quantities $\phi_{\mathbf{B r} \pm}^{2}$ are not proportional to the energy coupling factors $\beta_{L \pm}$ [see Eq. (7)], but to the Brownian noise coupling factors

$$
\beta_{\mathrm{Br} \pm}=\frac{\frac{1}{2} L_{\mathrm{in}} I_{ \pm}^{2}}{k T}=\frac{L_{\mathrm{in}} \phi_{\mathrm{Br} \pm}}{2 M^{2} k T},
$$

because the signal originates only in the bar, while the Brownian noise comes from both oscillators. In our detector, when the transducer is biased at $V_{b}=320 \mathrm{~V}$, we have, for $T=2.6 \mathrm{~K}$,

$$
\phi_{\mathrm{Br}-}=1.35 \times 10^{-4} \phi_{0}, \quad \phi_{\mathrm{Br}+}=0.97 \times 10^{-4} \phi_{0},
$$

with $\phi_{0}=2.068 \times 10^{-15} \mathrm{~Wb}$.

The spectral density of the Brownian noise $S_{\phi \mathrm{Br}}(\omega)$ has Lorentzian shape, at each of the modes, with a width given by the corresponding $Q$ factor. At the mode frequencies, we have

$$
S_{\phi \mathrm{Br}}\left(\omega_{ \pm}\right)=\phi_{\mathrm{Br} \pm}^{2} \tau_{ \pm}
$$

The wideband noise, which we can assume as being constant in the frequency range of observation, is characterized in terms of its spectral density $S_{0}$, which we usually express in units of magnetic flux in the SQUID loop. For our SQUID the theoretical value of this quantity is $\sim 4-5 \times 10^{-7} \phi_{0} / \sqrt{\mathrm{Hz}}$ at $4.2 \mathrm{~K}[19]$; the measured value for an uncoupled isolated device is $1.2 \times 10^{-6} \phi_{0} / \sqrt{\mathrm{Hz}}$ at $4.2 \mathrm{~K}$ [17] and $\sqrt{S_{0}} \sim 3 \times 10^{-6} \phi_{0} / \sqrt{\mathrm{Hz}}$ for our SQUID amplifier (at $2.6 \mathrm{~K}$ ).

Here we remark that in addition to the fundamental 
"calculable" noise, due to known physical effects, we record, occasionally, some excess noise of nonstationary nature, which shows up as pulses as well as spurious resonance peaks. (One peak which exhibits memory effects is due to a mechanical resonance of the antenna-transducer system.)

\section{Sensitivity}

The bandwidth of the signal, which is the same as that of the narrow-band noise, is determined for each of the two modes by the corresponding $Q$ factor

$$
\Delta v_{ \pm}=\frac{v_{ \pm}}{Q_{ \pm}}
$$

with typical values in the millihertz range.

The bandwidth of the signal-to-noise ratio (SNR), that is, the actual observation bandwidth of the detector, is much larger. This is determined by the "crossover" frequencies where the amplitudes of the spectrum of the narrow-band noise become equal to that of the wideband noise. For each of the two modes, we have

$$
\Delta v_{\mathrm{SNR} \pm}=\Delta v_{ \pm}\left(\frac{1+\Gamma_{ \pm}}{\Gamma_{ \pm}}\right)^{1 / 2},
$$

where the quantities

$$
\Gamma_{ \pm}=\frac{S_{0}}{S_{\phi \mathrm{Br} \pm}}
$$

represent the ratios between the spectra of the wideband and narrow-band noise (at the resonance peaks) and depend on the $Q$ factors of the detector [see Eq. (11)]. Since the spectral ratios $\Gamma_{ \pm}$are usually several orders of magni- tude smaller than unity, the SNR bandwidth is much larger than the signal bandwidth: In our case, it is indeed in the hertz range.

The sensitivity to a short burst of GW's is maximized by filtering the data using algorithms aimed at the detection of sudden variations of the energy of the modes. These variations can be assumed to be due to equivalent forces acting on the modes; these forces are the quantities that are actually estimated by the filter from the observed data. Therefore the outputs of the filter, in the absence of any signal or external disturbance, are sequences of estimates of the Nyquist noise forces.

The performance of a filtering algorithm is given by the mean value of the energy of the filtered noise referred to the antenna. We express this value as a temperature (dividing it by the Boltzmann constant $k$ ) to obtain an immediate comparison with the unfiltered noise that corresponds closely to the thermodynamic operating temperature. When using an optimum filter, these effective temperatures are

$$
T_{\text {eff } \pm}=\frac{4}{a_{ \pm}} T\left(\frac{\Gamma_{ \pm}}{1+\Gamma_{ \pm}}\right)^{1 / 2} \simeq \frac{4}{a_{ \pm}} T \sqrt{\Gamma_{ \pm}},
$$

where the coefficients $a_{ \pm}$take into account the partition between the two modes of the energy of the signal ( $a_{+}=a_{-}=0.5$ in the case of perfect tuning) and $T$ is the temperature of the bar (more precisely, here $T$ is the equivalent temperature of the narrow-band noise, which includes the back-action effects; these are, however, negligible in our detector). With $V_{b}=320 \mathrm{~V}$, we have $a_{-}=0.322, a_{+}=0.678$.

\begin{tabular}{|c|c|c|}
\hline \multicolumn{2}{|l|}{$\begin{array}{l}\text { Frequency of the bar } \\
\text { (first longitudinal mode) }\end{array}$} & $v_{b}=915.69 \mathrm{~Hz}$ \\
\hline Mass of the bar & & $M=2270 \mathrm{~kg}$ \\
\hline $\begin{array}{l}\text { Frequency of the transducer } \\
\text { (first flexural mode) }\end{array}$ & & $v_{t 0}=917.95 \mathrm{~Hz}$ \\
\hline Mass of the transducer (disk) & & $M_{t}=0.40 \mathrm{~kg}$ \\
\hline Ratio of the two equivalent masses & & $\mu=3.03 \times 10^{-4}$ \\
\hline Capacity of the transducer & & $C_{t}=3890 \mathrm{pF}$ \\
\hline Gap of the transducer & & $d=52 \mu \mathrm{m}$ \\
\hline Electrical field in the transducer & $\left(V_{b}=320 \mathrm{~V}\right)$ & $E=6.15 \times 10^{6} \mathrm{~V} / \mathrm{m}$ \\
\hline Frequency of the modes & $v_{-}=904.7 \mathrm{~Hz}$ & $v_{+}=921.3 \mathrm{~Hz}$ \\
\hline Merit factors of the modes & $Q_{-}=0.77 \times 10^{6}$ & $Q_{+}=1.01 \times 10^{6}$ \\
\hline Decay times of the modes & $\tau_{-}=271 \mathrm{~s}$ & $\tau_{+}=349 \mathrm{~s}$ \\
\hline Energy coupling factors & $\beta_{L-}=1.6 \times 10^{-4}$ & $\beta_{L+}=2.2 \times 10^{-4}$ \\
\hline Transformer primary inductance & & $L_{0}=2.5 \mathrm{H}$ \\
\hline Transformer secondary inductance & & $L_{s}=1.6 \mu \mathrm{H}$ \\
\hline Transformer coupling constant & & $k_{t}=0.77$ \\
\hline dc SQUID input coil inductance & & $L_{\text {in }}=1.0 \mu \mathrm{H}$ \\
\hline dc SQUID flux noise (unilateral) & & $\sqrt{S_{0}}=3 \times 10^{-6} \phi_{0} / \sqrt{\mathbf{H z}}$ \\
\hline Modulation frequency of the dc SQUID & & $v_{m}=80.1 \mathrm{kHz}$ \\
\hline Brownian fluxes $(T=2.6 \mathrm{~K})$ & $\phi_{\mathrm{Br}-}=1.35 \times 10^{-4} \phi_{0}$ & $\phi_{\mathrm{Br}+}=0.97 \times 10^{-4} \phi_{0}$ \\
\hline Spectral ratios & $\Gamma_{-}=0.92 \times 10^{-6}$ & $\Gamma_{+}=1.4 \times 10^{-6}$ \\
\hline SNR bandwidths & $\Delta v_{\mathrm{SNR}-}=1.22 \mathrm{~Hz}$ & $\Delta v_{\mathrm{SNR}+}=0.78 \mathrm{~Hz}$ \\
\hline
\end{tabular}

The filtered sequences, obtained from the observation of the two modes, are usually combined together in a sin-

TABLE I. Characteristics of the experimental apparatus. 
gle sequence. Each sample of the new sequence is obtained by selecting the minimum value between the two corresponding (i.e., taken simultaneously) samples of the mode sequences. This criterion is discussed in Sec. IV B 1 . Since the data have exponential distribution, it can be shown that the effective temperature of the resulting sequence is

$$
T_{\text {eff }}^{-1}=T_{\text {eff }-}^{-1}+T_{\text {eff }+}^{-1} .
$$

At $T=2.6 \mathrm{~K}$, using the parameters of Table $\mathrm{I}$ and the values of $a_{ \pm}$given above, we have

$$
T_{\text {eff }-}=31 \mathrm{mK}, T_{\text {eff }+}=18 \mathrm{mK}, T_{\text {eff }}=11 \mathrm{mK},
$$

the difference between $T_{\text {eff }-}$ and $T_{\text {eff }+}$ arising also from the $Q$ values of the modes.

By substituting $T_{\text {eff }}$ in Eq. (1), we have $H=8.5 \times 10^{-22}$ $\mathrm{s}$ and $h=8.5 \times 10^{-19}$ under the assumption of bursts with duration of $1 \mathrm{~ms}$.

\section{Calibration of the detector}

During December 1989, the detector was calibrated by means of the sinusoidal gravitational near field generated by a small rotating quadrupole [22].

The center of mass of the rotor, with quadrupole moment $5.55 \times 10^{-3} \mathrm{~kg} \mathrm{~m}^{2}$, was at $3.52 \pm 0.05 \mathrm{~m}$ from that of the antenna. Its angular velocity was equal to one-half of the frequency of one antenna mode. In these conditions the amplitude of the sinusoidal equivalent force applied to the bar was $2.19 \times 10^{-11} \mathrm{~N}$.

This experiment was performed with the antenna equipped with a FET amplifier and with a relatively low value of the field in the transducer $\left(4.5 \times 10^{5} \mathrm{~V} / \mathrm{m}\right)$. The reason was to reduce the loss of electrical charge from the transducer capacitor in order to keep within few tens of microhertz the variations of the frequencies of the modes during the measurements, since each run had to last longer than $1 \mathrm{~h}\left(Q \simeq 6 \times 10^{6}, \tau \simeq 34 \mathrm{~min}\right)$ to observe the rise of the oscillations from the background to a steady-state value.

The measured value of the oscillations, observed through a lock-in amplifier driven at the frequency of the mode which was excited by the rotor, was well above the Brownian noise and in very good agreement (about $20 \%$ in energy) with the result of the computations.

Another calibration was performed during December 1991, with the detector operating in standard conditions, i.e., with the SQUID amplifier and high value of the field in the transducer. This time the force, a wave packet, was applied using a small piezoelectric transducer (PZT) ceramic glued on the bar.

The PZT ceramic was first calibrated, at each of the modes, by determining the value of the constant $C_{1}$ of the electrical equivalent circuit of the antenna [23] as seen at the electrical port of the PZT ceramic. We found $C_{1-}=2.61 \times 10^{-20} \mathrm{~F}, C_{1+}=8.39 \times 10^{-20} \mathrm{~F}$ with a relative standard deviation of $5 \%$. This was obtained by applying to the PZT ceramic a wave packet at the frequency of the mode, of known amplitude and duration, and by observing the corresponding voltage response of the PZT ceramic itself when switched to a low-noise amplifier [24].

Afterwards, we applied to the PZT wave packets of amplitude $(\mathrm{rms}) V_{\mathrm{PZ}}$, duration $t_{\mathrm{PZ}}$, and frequency $v_{ \pm}$, and observed the response of the antenna at the output of the SQUID amplifier. The energy deposited on the bar was

$$
E_{ \pm}=\pi^{2} C_{1 \pm} v_{ \pm}^{2} V_{\mathrm{PZ}}^{2} t_{\mathrm{PZ}}^{2},
$$

with the parameter chosen to obtain values $\left(10^{3}-10^{4} \mathrm{~K}\right)$ well above the Brownian noise.

By comparing, at each of the modes, the experimental results $E_{\text {meas } \pm}$ with the corresponding values $E_{ \pm}$computed using the known parameters of the detector, we obtained the ratios $E_{-} / E_{\text {meas }}=1.18$ and $E_{+} / E_{\text {meas }}+$ $=1.40$, both with a relative standard deviation of $20 \%$.

We attribute the discrepancy to the imperfect knowledge of some parameters of the detector. We also note that the above values are consistent with the indication provided by the long-term averages of the Brownian noise of the antenna that seem to be slightly below the thermodynamic temperature.

We did not use the above figures to correct our experimental data because we plan to perform new calibrations with improved methods.

\section{ACQUISITION AND ANALYSIS OF THE DATA}

The data-acquisition system of the Explorer detector is based on a microVax 3800 minicomputer, which is connected to the international network (the data are transmitted to the Vax cluster located at the University of Rome La Sapienza) and can be remotely operated. This machine, in addition to the acquisition and permanent recording of the data in a form suitable for further off-line analysis, performs various functions of online analysis for diagnostic purposes.

The data are analyzed using three different techniques: the standard Wiener filter with fixed parameters (on line); an adaptive Wiener filter (off line), which accounts for the nonstationary nature of the noise, thereby improving the sensitivity; an adaptive matched filter (on line), which has been recently developed [25] and operates on the signal directly sampled at the output of the SQUID instrumentation (i.e., bypassing the lock-in amplifiers).

\section{A. Data acquisition, timing, and on-line analysis}

The analog-to-digital (AD) conversion and the acquisition of the data are performed inside the microVax by the ADV11 card with 16 input lines (see Table II). The timing signal that controls the conversion (and therefore sets the sampling time $\Delta t$ ) is obtained by dividing the $5-\mathrm{MHz}$ reference frequency of the rubidium clock of CERN. A complete scanning of the inputs is done at the rate of $220.07042 \mathrm{~Hz}$.

The first 12 channels (with the exception of channel 6) are subsampled before being recorded with sampling time $\Delta t=0.2908 \mathrm{~s}$. In order to minimize any disturbance due to aliasing effects, the outputs of the lock-in amplifiers (the first four channels) are filtered with two-pole low- 
TABLE II. Data-acquisition channels.

\begin{tabular}{ll}
\hline \hline CH 1 & $p_{-}(t)$ at $v_{-}$ \\
CH 2 & $q_{-}(t)$ at $v_{-}$ \\
CH 3 & $p_{+}(t)$ at $v_{+}$ \\
CH 4 & $q_{+}(t)$ at $v_{+}$ \\
CH 5 & calibration signal at $v_{c}(913 \mathrm{~Hz})$ \\
CH 6 & direct acquisition \\
CH 7 & noise at $v_{\mathrm{FB}}(909 \mathrm{~Hz})$ \\
CH 8 & accelerometer $0.2-100 \mathrm{~Hz}$ \\
CH 9 & accelerometer $900-930 \mathrm{~Hz}$ \\
CH 10 & search coil $900-930 \mathrm{~Hz}$ \\
CH 11 & clock (see text) \\
CH 12 & antenna output $20-70 \mathrm{~Hz}$ \\
CH 13 & pressure of helium bath \\
CH 14 & evaporation rate \\
CH 15 & pressure of isolation vacuum \\
\hline \hline
\end{tabular}

pass filters with a $5-\mathrm{Hz}$ cutoff frequency before the AD conversion.

Channel 6, called "direct acquisition" and used for the matched filter, provides the signal of the antenna (taken before that of the lock-in amplifier), which is filtered with an hardware bandpass filter with flat response between 902 and $926 \mathrm{~Hz}$.) After the AD conversion, the data of this channel are first bandpass filtered in software in the frequency range $900-927.5 \mathrm{~Hz}$ and are subsequently permanently recorded at the rate of $55 \mathrm{~Hz}$.

We also have three "slow" channels, which are recorded every $10 \mathrm{~s}$, which we use to monitor the liquid-He bath pressure (which is related to the antenna temperature), the evaporation rate, and the pressure in the isolation vacuum.

Each block of recorded data contains the timing of its first record from which we can derive the times of all the data of the block. This timing information is obtained from a radio receiver tuned to the $H B G$ time standard broadcasting station (Pragins, $\mathrm{CH}, 75 \mathrm{kHz}$ ). The quality of this information is continuously checked by comparing it with that derived from the rubidium clock of CERN: The $1-\mathrm{Hz}$ pulses from the two clocks are added up and applied to the input of channel 11. The overall absolute accuracy is, at present, within $10-20 \mathrm{~ms}$ because of the internal architecture of the Vax system.

Our data-acquisition software also provides monitoring and diagnostic facilities for the operation of the detector by performing several on-line analysis functions. The recorded data can, in fact, be displayed in the form of time histories, distributions, and spectra. Moreover, the data representing the observed modes are processed with a fixed-parameter Wiener filter and the data of channel 6 with an adaptive matched filter, both operating in real time. All the results (statistics, time histories, and distributions) can be displayed, thereby providing a powerful diagnostic tool for managing the continuous operation of the experiment.

A systematic search for "events" (that is, data samples with values exceeding suitable thresholds, defined in terms of standard deviations) is also performed on line for all the recorded channels as well as for the outputs of the above filters; these events are in turn permanently recorded.

The program controls the frequency setting of the synthesizers used as reference for the lock-in amplifiers at the frequencies of the modes $v_{-}$and $v_{+}$in order to track any variation of these frequencies. This is done every $2 \mathrm{~h}$ on the basis of the frequencies estimated from a fit of the Fourier transform of the recorded data.

\section{B. Analysis of the data}

The analysis consists in processing (a) the data of channels 1-4 corresponding to the two components of the modes $v_{-}$and $v_{+}$, obtained from the lock-in amplifiers and recorded with $\Delta t=0.2908 \mathrm{~s}$, and (b) the data of channel 6 , direct acquisition, which are recorded with $\Delta t=18.18 \mathrm{~ms}$.

The data of channels $1-4$ are first normalized to the actual SQUID gain, using the information provided by the calibration channel (5) (averaged over $2 \mathrm{~min}$ ), and then scaled to express the vibrations of the modes of the detector, using the known parameters of the detector (gain of the electronics, energy coupling factors, etc.). The sum of the squares of these components, for each of the modes, represents the vibration energy, which is expressed in kelvin: The average values of these quantities are estimates of the temperature of the modes.

The filtering of the data is performed in software by means of optimum filters aimed at the detection of short bursts of gravitational radiation. These signals are discriminated from the noise on the basis of their different statistical properties: A signal gives rise to a sudden variation of the status of the modes in $p, q$ plane, while in the same frame of reference the narrow-band noise gives very slow variations (with correlation times $\left.\tau_{ \pm}\right)$.

The filters used at present are described in the following sections: Two of them are applied to the data in real time, and another is applied off line to the archived data.

\section{Wiener-Kolmogorov filter}

The Wiener-Kolmogorov (WK) filter [26,27] (which is used for the on-line analysis) assumes the noise as only due to the calculable contributions discussed in Sec. III B, i.e., as a superposition of white and Lorentzian noise, and requires a knowledge of the spectral ratio $\Gamma$ and the decay time constant $\tau$ for each mode. This filter cancels, first, the dynamics of the antenna and the lock-in amplifiers (eliminating the corresponding delays) and then smooths the noise with the time constant

$$
\tau_{3 \pm}=\tau_{ \pm}\left(\frac{\Gamma_{ \pm}}{1+\Gamma_{ \pm}}\right)^{1 / 2},
$$

according to the transfer function

$$
W_{ \pm}(j \omega)=\frac{1}{1+\Gamma_{ \pm}} \frac{1}{W_{1 \pm} W_{2}} \frac{\omega^{2}}{\tau_{3 \pm}^{-2}+\omega^{2}}
$$

where $W_{1 \pm}$ is the transfer function of the antenna (taking into account the demodulation performed by the lock-in 
amplifiers) and $W_{2}$ the transfer function of the integrators contained in the lock-in amplifiers [27]:

$$
W_{1 \pm}(j \omega)=\frac{1}{1+j \omega \tau_{ \pm}}, \quad W_{2}(j \omega)=\frac{1}{1+j \omega t_{0}} .
$$

This is done for each of the two modes by constructing two sequences,

$$
\begin{aligned}
& w_{p}(j)=\sum_{k=-n}^{n} p(j-k) w(k), \\
& w_{q}(j)=\sum_{k=-n}^{n} q(j-k) w(k),
\end{aligned}
$$

from the two components of the mode signal, $p(t)$ and $q(t)$, recorded at time intervals $\Delta t$; the coefficients $w(k)$ are the weights of the filter, which are obtained from the inverse Fourier transform of the above function $W(j \omega)$; the sums are limited to the interval $-n,+n$, outside which the contribution of the weights is negligible.

The filter response to a burst is a two-sided exponential function with time constant $\tau_{3 \pm}$, with its peak at the time of occurrence of the input impulse.

At each sampling instant $j \Delta t$, the sum of the squares of the filtered components of each mode represents an estimate of the variation of the vibration energy of the antenna:

$$
\begin{aligned}
& \rho_{-}^{2}(j)=K_{-}\left[w_{p-}^{2}(j)+w_{q-}^{2}(j)\right], \\
& \rho_{+}^{2}(j)=K_{+}\left[w_{p+}^{2}(j)+w_{q+}^{2}(j)\right],
\end{aligned}
$$

where $K_{-}$and $K_{+}$are normalization constants. These constants also account for the partition between the modes of the energy deposited by the input burst.

The corresponding average values

$$
T_{\text {eff- }}=\left\langle\rho_{-}^{2}(j)\right\rangle, \quad T_{\text {eff }+}=\left\langle\rho_{+}^{2}(j)\right\rangle
$$

represent the fluctuation energy of the residual noise, that is, the effective temperatures of the antenna as observed from the modes.

We usually combine together the estimates obtained from the observation of the two modes with the rule

$$
\rho^{2}(j)=\min \left(\rho_{-}^{2}(j), \rho_{+}^{2}(j)\right) .
$$

The average value of this quantity provides the combined effective temperature $T_{\text {eff }}$, given by Eq. (20). This rule, considering the data as representing estimates of a signal, is based on the assumption that a short burst (with a flat spectrum in the frequency range of the modes) deposits known fractions of energy in the two modes. The two corresponding estimates $\rho_{-}^{2}$ and $\rho_{+}^{2}$ should therefore be equal.

As an alternative, we also perform a weighted average of the two estimates:

$$
\overline{\rho^{2}}(j)=\frac{T_{\mathrm{eff}+} \rho_{-}^{2}(j)+T_{\mathrm{eff}-\rho_{+}^{2}(j)}}{T_{\mathrm{eff}+}+T_{\mathrm{eff}-}}
$$

Note that the above formulas (28) and (29) only hold for signals somewhat larger than the background [from the estimates (25) and (26), one should in fact subtract the mean values of the background].

\section{Wiener-Kolmogorov adaptive filter}

The adaptive version of the above filter (which is used for the analysis performed off line) operates similarly, but it requires no assumption as regards the noise.

The weights of the filter to be applied to the data during a given period of time (typically $2 \mathrm{~h}$ ) are, in fact, derived from an estimate of the actual spectrum of the noise during the same time period. Its actual implementation is based on frequency domain operations: The filtered data are obtained from the inverse Fourier transform of the product between the Fourier transform of the data and the transfer function of the filter.

Its performance is very close to that of the nonadaptive WK filter when the noise is well behaved. In the presence of spurious disturbances, whose spectral distribution may also change with time, it provides a considerable improvement [2].

\section{Adaptive matched filter}

The adaptive matched filter is applied in real time to the data of channel 6 , which are sampled at high speed $(55 \mathrm{~Hz})$. It is based on an approach [25] different from that of the filters described above, which is more general in the sense that it can be used for detecting signals of any given shape; in the case of an impulsive input signal, as in the present implementation, its operation and the results it provides are equivalent to those of a Wiener filter. In practice, however, it performs better, since it does not suffer of the bandwidth limitations of the Wiener filters (due to the relatively slow sampling time of the data we use to limit the amount of data recorded).

The adaptive matched filter processes the data of channel 6 in the frequency domain according to the transfer function $[25,28]$

$$
M(j \omega)=\frac{K F^{*}(j \omega)}{S_{n}(\omega)},
$$

where $F^{*}(j \omega)$ is the complex conjugate of the transfer function of the antenna (here a resonant function since the data are not demodulated), $S_{n}(\omega)$ is the spectrum of the noise, and $K$ is a normalization constant which provides the squared output in units of kelvin.

This filter is adaptive in the sense that the spectrum $S_{n}(\omega)$ is obtained from the estimation of the actual noise of the data during the last $2 \mathrm{~h}$ (by averaging 200 periodograms). A plot of the magnitude versus frequency of an actual implementation of this filter is shown in Fig. 4 along with the corresponding spectrum.

Note that since this filter is computed every $2 \mathrm{~h}$ and operates in real time, the estimation of the noise and construction of the adaptive filter is performed on the data of the previous time period. As a consequence, its performance may be seriously degraded if the noise spectrum is highly nonstationary. 


\section{EXPERIMENTAL RESULTS}

We present here the experimental results obtained with the Explorer detector during the period July 1990 to December 1991, with the cryostat operated in superfluid helium regime: Typically at $1.8 \mathrm{~K}$ with a pressure of 20 mbar on the helium bath (the cryostat was let up to at 4.2 $\mathrm{K}$ only during the December period). The temperature of the antenna was stabilized at $T \simeq 2.6 \mathrm{~K}$ from December 1990.

The transducer is charged by means of a battery that is then disconnected. We charged it on 18 June 1990 at $-316 \mathrm{~V}$ and a few other times at similar voltages. By observing the drift of the mode frequencies (we obtained, in particular, $\delta v_{-}=67 \mathrm{mHz}, \delta v_{+}=40 \mathrm{mHz}$ during the month of July 1990 and $\delta v_{-}=29 \mathrm{mHz}, \delta v_{+}=17 \mathrm{mHz}$ during the month of November 1990), we found that the transducer loses its electrical charge at a rate of about 70 $\mathrm{mV} / \mathrm{day}$, corresponding to an insulation resistance of about $3 \mathrm{~T} \Omega$.

The performance of Explorer during the period July 1990 to December 1991 is summarized in Fig. 5. Here we have plotted the hourly values of the minimum detectable metric tensor perturbation $h$ derived by means of Eq. (1) under the conventional assumption of bursts with duration of $1 \mathrm{~ms}$. The values of $T_{\text {eff }}$ used to compute $h$ are the hourly averages of the data of the two modes filtered off line with the adaptive Wiener filter and combined with the rule of Eq. (28). The periods when no data are reported were used for refilling with cryogenic liquids and for
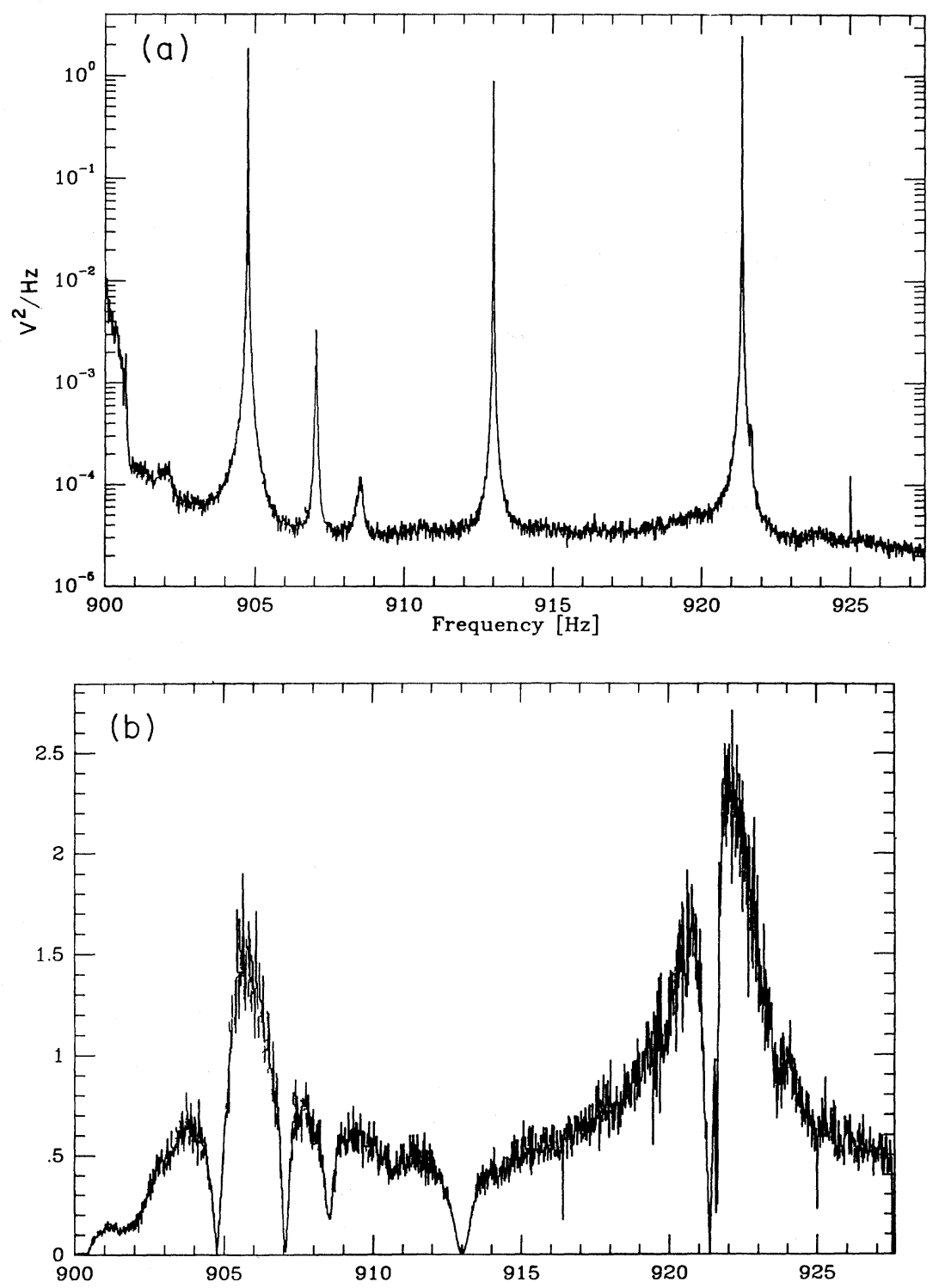

FIG. 4. (a) Spectrum of the data from channel 6 (direct acquisition of the two modes): $2 \mathrm{~h}$ during 9 May 1991 . (b) Frequency response of the adaptive matched filter corresponding to spectrum (a): The response decreases where there are spurious peaks in the data (including the calibration line at $913 \mathrm{~Hz}$ ). 
performing various tests. Overall, the antenna was recording data for more than $\frac{2}{3}$ of the total time (we plan to increase this duty cycle to about $80 \%$ by increasing the time between the refills). We note that most of the time the sensitivity was better than $h \approx 10^{-18}$, a major improvement with respect to our past performance [13]. It is the first time that a GW antenna operated for such a long period of time with this sensitivity, about 1000 times better in energy (and $\sqrt{1000}$ in amplitude) than that of the original room-temperature antennas.

From June 1991 Explorer is taking data in coincidence with the LSU antenna, which had begun to operate, and work is in progress for the coincidence analysis.

In Fig. 5 we have reported all data recorded by the system, excluding only those recorded while the operators were disturbing the apparatus (during tests, during cryogenic refills, etc.). But is is possible to improve the
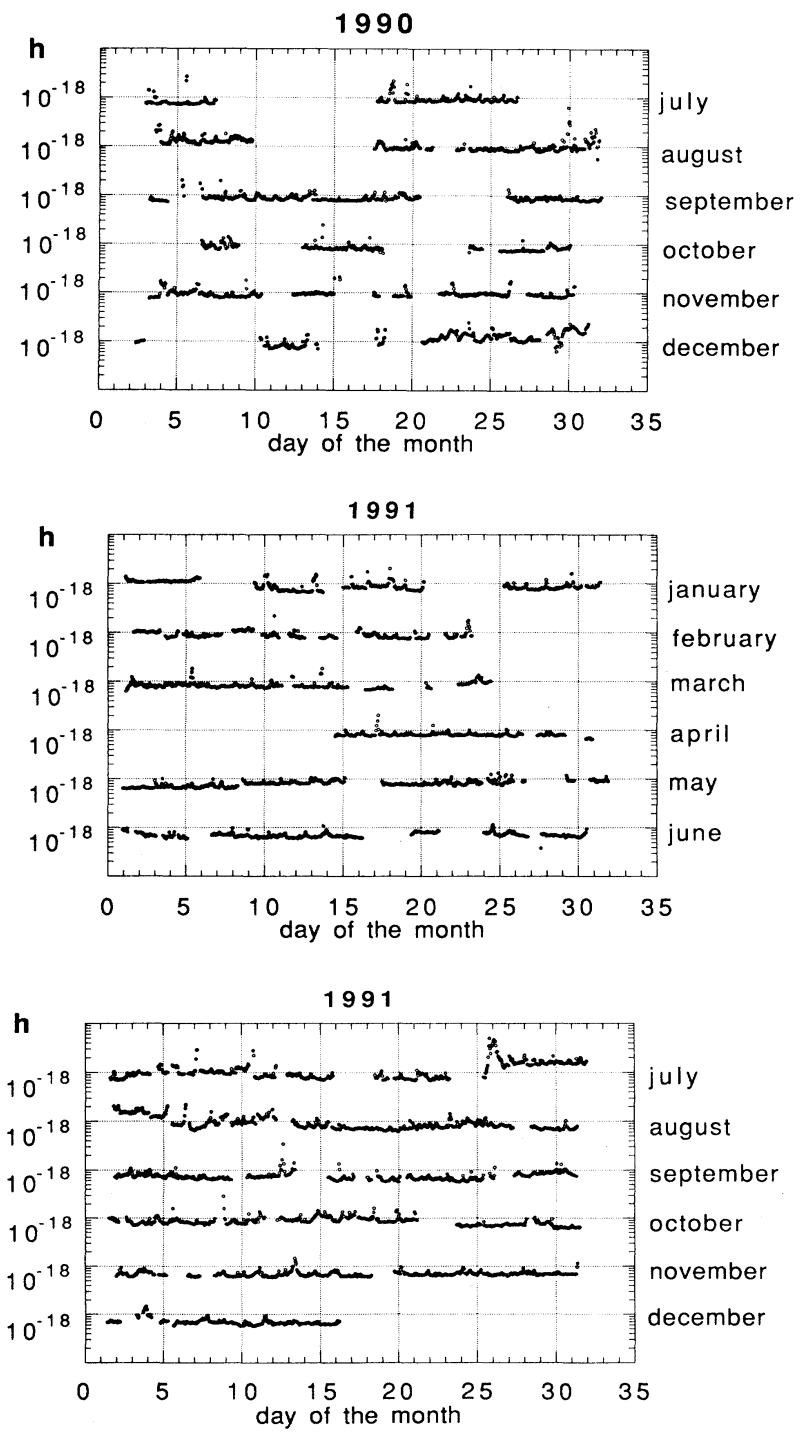

FIG. 5. Hourly values of the minimum detectable metric tensor perturbation $h$ (July 1990 to December 1991).

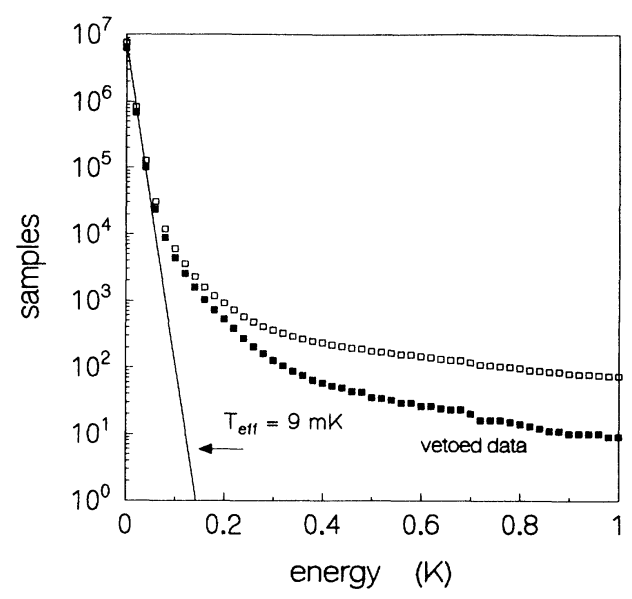

FIG. 6. Distributions of the data recorded during November $1991(609 \mathrm{~h})$ : all the data and vetoed data.

signal-to-noise ratio of any data analysis by eliminating the data that are clearly correlated with the auxiliary sensors, such as the seismic and electromagnetic sensors, or show clear signs of improper functioning of the apparatus. In practice, we do not throw away these "bad" data; we just put a mark on them, since they may contain useful information. We use the following procedure for "cleaning" the data: We accept only the data [filtered with the adaptive Wiener filter and combined with the rule of Eq. (28)] that are not included in time windows of $\pm 5 \mathrm{~s}$ centered at the times when the auxiliary channels $(8-12)$ indicate the possible presence of a disturbance. This amounts to eliminating $\sim 11 \%$ of the data. The events selected in this way are marked with a minus sign and recorded on a file for possible use in coincidence analysis.

The event distributions obtained with the above selection criteria are shown in Fig. 6 for a time period of 609 h, from 2 November 1991, 02:00 UT to 31 November 1991, 12:00 UT. The upper curve represents all the data (no auxiliary vetos are considered). The second curve includes the above-mentioned selection with the auxiliary channels. Finally, the straight line is the fit of the data points smaller than $50 \mathrm{mK}$ and its slope gives $T_{\text {eff }}$. It is clear that $T_{\text {eff }}$ alone does not represent the sensitivity of the antenna, as the tail of the distribution is the most important when considering possible signals, with and without coincidence with another antenna (which, in all cases, are essential).

In the last week of 1991, the detector has been warmed up to perform a "tune up" of the cryogenic system.

\section{A. Upper limit on GW pulses}

Figure 7 shows the integral distribution of all the "events" which have peak values above $100 \mathrm{mK}$ and do not coincide with local disturbances, recorded from 2 June 1991 to 16 December 1991. These events have been obtained from the data of the two modes $\left[\rho_{ \pm}^{2}(i)\right]$ by using the combination rule of Eq. (28), i.e., by selecting the minimum and then by taking the peak values of this se- 


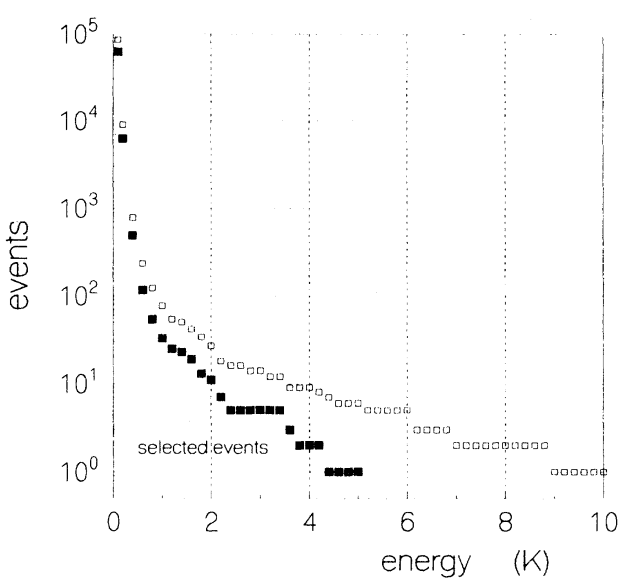

FIG. 7. Distributions of all the events with peak values above $100 \mathrm{mK}$ that do not coincide with local disturbances (from 2 June 1991 to 16 December 1991): all the events and only the events such that the ratio between the energies of the two modes is in the range from $\frac{1}{2}$ to 2 .

quence, in order to consider isolated pulses. The total observation time is 133.8 days.

Figure 7(b) also shows the distribution of those events such that the ratio $R$ between the two modes $\left[\rho_{+}^{2}(i) / \rho_{-}^{2}(i)\right]$ is between $\frac{1}{2}$ and 2 . This requirement is certainly fulfilled by any short burst of GW's, even taking into account the noise.

Without performing coincidences with the data of another detector of comparable sensitivity, it is not possible to derive any positive conclusion from the above distributions. The presence of many events that are clearly in excess with respect to the statistics of the low-level background may, in fact, be due to various causes: external disturbances not vetoed by the auxiliary channels, acoustic emission effects in those parts of the mechanical suspensions system that are highly stressed, etc.

However, the distributions of the events of Fig. 7 may be used to establish an upper limit on the rate and strength of the GW pulses during the observation time of our detector. The analysis has been performed following the method reported in [10], i.e., assuming sources with random polarization and isotropic distribution over the celestial sphere and assuming, in the statistical analysis, probabilities of false detection and false dismissal both equal to 0.05 (corresponding to a confidence level of 95\%).

The results of the analysis are plotted in the two lower curves of Fig. 8: the lowest obtained with the events of Fig. 7 with energy ratio of the modes between $\frac{1}{2}$ and 2, and the other one with all the events. In the same figure, we have also plotted the upper limits obtained with the Stanford detector during 1982 [8], with an observation time of 73.7 days, and with the Rome and Stanford detec-

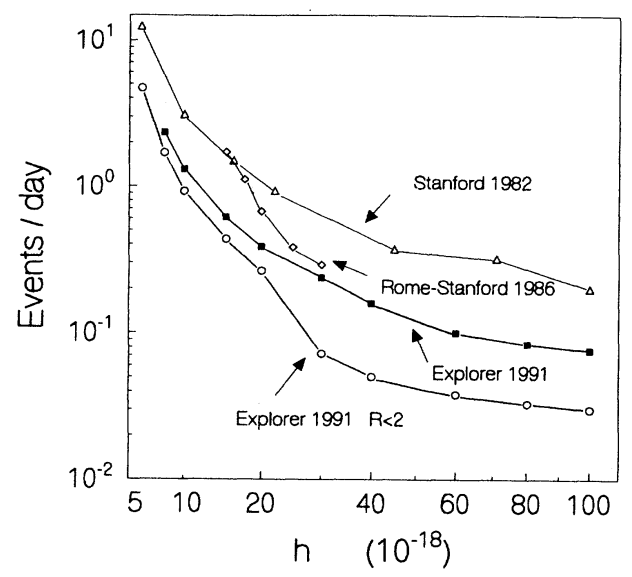

FIG. 8. Upper limit for the rate and strength of GW pulses during 133.8 days of observation, compared with the results obtained by Stanford (1982) and by the Stanford and Rome detectors in coincidence (1986).

tors in coincidence during 1986 [10], with an observation time of 35.5 days.

\section{CONCLUSIONS}

The results of the operation of the Explorer detector during 1990 and 1991, as well as those of the LSU detector since June 1991 [29], show that the cryogenic gravitational wave antennas of the second generation are ready to provide good quality data over long times, with much better sensitivity than in the past: more than three orders of magnitude in energy, compared with the detectors of the first generation which operated at room temperature.

The data of a single detector, however, can only be used to place new upper limits on the rate and strength of gravitational radiation pulses during the observation period. Indeed, they cannot be used to derive any conclusion about the real nature of any observation of burst events, i.e., to distinguish between $\mathrm{GW}$ events and noise. Only simultaneous coincidence observations with remote detectors, such as the Explorer in Geneva and the LSU detector in Baton Rouge, Louisiana, can hope to do this.

At present, in fact, the data collected by these detectors from June to December 1991 have been exchanged between the two groups, which are working in close collaboration, and are being analyzed, searching for coincidence events.

\section{ACKNOWLEDGMENTS}

We thank Dr. Ph. Bernard for his advice and collaboration and F. Bronzini for his contribution to the design of the cryostat. We thank Professor W. O. Hamilton for his help in obtaining for us the aluminum bar. We also thank G. Federici, G. Martinelli, and E. Serrani for their valuable technical support and M. Rieubland for the very efficient supply of cryogenic liquids. 
[1] B. F. Schutz, in Fourteenth Texas Symposium on Relativistic Astrophysics, Proceedings, Dallas, Texas, 1988, edited by E. J. Fenyves [Ann. N.Y. Acad. Sci. 571, 27 (1990)].

[2] E. Amaldi, P. Astone, M. Bassan, P. Bonifazi, M. G. Castellano, G. Cavallari, E. Coccia, C. Cosmelli, S. Frasca, E. Majorana, I. Modena, G. V. Pallottino, G. Pizzella, P. Rapagnani, F. Ricci, M. Visco, and Zhu Ning, Europhys. Lett. 12, 5 (1990).

[3] R. P. Giffard, Phys. Rev. D 14, 2478 (1976).

[4] C. M. Caves, K. S. Thorne, R. W. P. Drever, V. D. Sandberg, and M. Zimmermann, Rev. Mod. Phys. 52, 341 (1980).

[5] G. V. Pallottino and G. Pizzella, Nuovo Cimento 4C, 237 (1981).

[6] P. F. Michelson, T. Aldcroft, J. Chiang, D. DeBra, J. Henderson, L. Mann, D. McKenzie, F. McLoughlin, H. J. Paik, R. Penny, J. C. Price, T. Stevenson, B. Vaughan, and C. Zhou, in Gravitational Astronomy: Instrument Design and Astrophysical Prospects, edited by D. E. McClelland and H.-A. Bachor (World Scientific, Singapore, 1991), p. 14.

[7] P. Astone, M. Bassan, P. Bonifazi, M. G. Castellano, E. Coccia, C. Cosmelli, S. Frasca, E. Majorana, I. Modena, G. V. Pallottino, G. Pizzella, P. Rapagnani, F. Ricci, and M. Visco, Europhys. Lett. 16, 231 (1991).

[8] S. P. Boughn, W. M. Fairbank, R. P. Giffard, J. N. Hollenhorst, E. R. Mapoles, M. S. McAshan, P. F. Michelson, H. J. Paik, and R. C. Taber, Astrophys. J. 261, L19 (1982).

[9] W. O. Hamilton, B.-X. Xu, W. Johnson, N. Solomonson, O. Aguiar, B. Price, J. Harper, G. Griffin, and D. Duncan, in Proceedings of the 5th Marcel Grossmann Meeting on General Relativity, Perth, Australia, 1988, edited by D. G. Blair and M. J. Buckingham (World Scientific, Singapore, 1989), p. 1701.

[10] E. Amaldi et al., Astron. Astrophys. 216, 325 (1989).

[11] P. Astone, M. Bassan, P. Bonifazi, E. Coccia, C. Cosmelli, S. Frasca, E. Majorana, I. Modena, G. V. Pallottino, G. Pizzella, P. Rapagnani, F. Ricci, and M. Visco, Cryogenics 32, 668 (1992).
[12] E. Amaldi et al., Nuovo Cimento 7C, 338 (1984).

[13] E. Amaldi et al., Nuovo Cimento 9C, 829 (1986).

[14] F. Bronzini, E. Coccia, I. Modena, P. Rapagnani, and F. Ricci, Cryogenics 25, 234 (1985).

[15] P. Rapagnani, Nuovo Cimento 5C, 385 (1982).

[16] Y. Ogawa and P. Rapagnani, Nuovo Cimento 7C, 21 (1984).

[17] C. Cosmelli, M. G. Castellano, and P. Carelli, in SQUID '91, Proceedings of the 4th International Conference, Berlin, Germany, 1991, edited by H. Koch and H. Lübbig (Springer-Verlag, Berlin, 1992).

[18] G. V. Pallottino and T. Lupi, Rev. Sci. Instrum. 61, 2436 (1990).

[19] J. Clarke, Proc. IEEE 77, 1208 (1989).

[20] M. G. Castellano and C. Cosmelli, J. Appl. Phys. 63, 2015 (1988).

[21] M. G. Castellano and C. Cosmelli, Nuovo Cimento 7C, 9 (1984).

[22] P. Astone et al., Z. Phys. C 50, 21 (1991).

[23] G. V. Pallottino and G. Pizzella, Nuovo Cimento 45B, 275 (1978).

[24] S. Boughn, M. Bassan, W. M. Fairbank, R. P. Giffard, P. F. Michelson, J. C. Price, and R. C. Taber, Rev. Sci. Instrum. 61, 1 (1990).

[25] S. Frasca, G. V. Pallottino, and G. Pizzella, in Proceedings of the 3rd European Signal Processing Conference, The Hague, The Netherlands, 1986, edited by I. T. Young (North-Holland, Amsterdam, 1986), p. 597.

[26] A. Papoulis, Probability, Random Variables, and Stochastic Processes (McGraw-Hill, New York, 1965).

[27] P. Bonifazi, S. Frasca, G. V. Pallottino, G. Pizzella, and V. Ferrari, Nuovo Cimento 1C, 465 (1978).

[28] A. D. Whalen, Detection of Signals in Noise (Academic, New York, 1971).

[29] W. O. Hamilton, Z. Geng, W. W. Johnson, E. Maucelli, S. Merkowitz, B. Price, N. Solomonson, and N. Zhu, presented at the 13th International Conference on General Relativity and Gravitation, Cordoba, Argentina, 1992. 


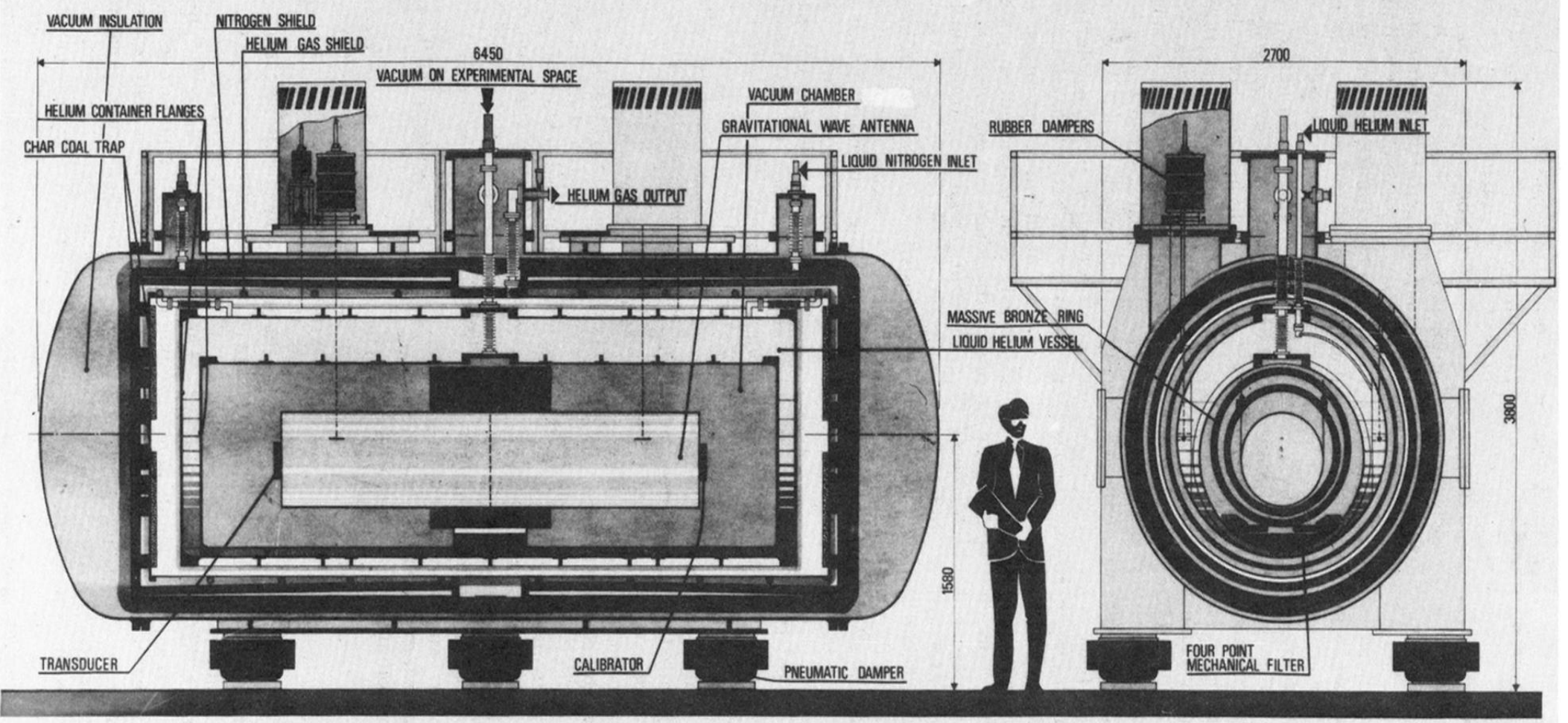

FIG. 1. Schematic diagram of the Explorer detector. 